\title{
Endoscopic Ultrasound-Guided Fine Needle Aspiration Using a 22-G Needle for Hepatic Lesions: Single-Center Experience
}

\author{
Ebru Akay', Deniz Atasoy ${ }^{2}$, Engin Altınkaya ${ }^{3}$, Ali Koç ${ }^{4}$, Tamer Ertan $^{5}$, Hatice Karaman ${ }^{1}$ and Erkan Caglar ${ }^{6}$ \\ ${ }^{1}$ Department of Pathology, Kayseri Training and Research Hospital, Kayseri, ${ }^{2}$ Department of General Surgery, Medicana Çamlıca \\ Hospital, Istanbul, ${ }^{3}$ Department of Gastroenterology, Cumhuriyet University, Faculty of Medicine, Sivas, ${ }^{4}$ Department of Radiology, \\ Kayseri Training and Research Hospital, Kayseri, ${ }^{5}$ Department of General Surgery, Kayseri Training and Research Hospital, Kayseri, \\ ${ }^{6}$ Department of Gastroenterology, Kayseri Training and Research Hospital, Kayseri, Turkey
}

Background/Aims: Endoscopic ultrasound-guided fine needle aspiration (EUS-FNA) has been accepted as a reliable tool in diagnosing and staging intra-abdominal tumors. In this study, we aimed to investigate the performance of EUS-FNA in the evaluation of liver masses and its impact on patient management and procedure-related complications retrospectively.

Methods: Data of patients who underwent EUS-FNA biopsies due to liver masses between November 2017 and July 2018 were retrieved retrospectively. Biopsies were performed using 22-G needles. The demographics, EUS-FNA results, sensitivity and specificity of the procedure, negative predictive value, positive predictive value, and specimen sufficiency rates were assessed.

Results: A total of 25 patients (10 females) were included in the study. The mean age was $62.73 \pm 15.2$ years. The mean size of the masses was $34.50 \pm 16.04 \mathrm{~mm}$. The technical success rate was $88 \%$. During the EUS-FNA procedure, each patient had only one pass with $94.45 \%$ of aspirate sufficiency rate and $86.3 \%$ of biopsy sufficiency rate. The diagnostic accuracy rate was $86.3 \%$. There were no complications.

Conclusions: For the evaluation of liver masses, EUS-FNA using a 22-G needle with even one pass had high aspiration and biopsy success rates accompanied with high diagnostic accuracy rates. Clin Endosc 2021;54:404-412

Key Words: Clinical management; Diagnostic accuracy; Endoscopic ultrasound; Fine needle aspiration; Liver mass

\section{INTRODUCTION}

The diagnosis of hepatic masses is important in clinical decision-making and typically established through percutaneous pathways with ultrasound and/or computed tomography (CT)-guided tissue biopsy for histopathological examination. ${ }^{1}$ However, this procedure becomes challenging or impossible in small-sized lesions because of the difficulty in reaching the

Received: March 2, 2020 Revised: July 17, 2020

Accepted: July 28, 2020

Correspondence: Erkan Caglar

Current affiliation- Balıkesir Üniversitesi, Tıp Fakültesi, Gastroenteroloji Bölümü, Çă̆ış, Balıkesir 26362, Turkey

Tel: +90-532-3081963, Fax: +90-266-6121417, E-mail: dr_erkan799@yahoo.com ORCID: https://orcid.org/0000-0001-7246-0952

(c) This is an Open Access article distributed under the terms of the Creative Commons Attribution Non-Commercial License (http://creativecommons.org/ licenses/by-nc/3.0) which permits unrestricted non-commercial use, distribution, and reproduction in any medium, provided the original work is properly cited. lesions by percutaneous means and biliary or vascular structures intervening on the biopsy line. In these cases, endoscopic ultrasound (EUS) could be one of the best methods with its applicability and reliability. ${ }^{2,3}$ EUS has been used in cases where conventional CT or transabdominal ultrasonography (USG) fails and for the evaluation of intrahepatic lesions such as hepatocellular carcinoma (HCC) without typical radiological findings. ${ }^{4-7}$

The aim of this study was to evaluate the role of EUS-guided fine needle aspiration (EUS-FNA) in the diagnosis of liver masses. Its impact on patient management and procedure-related complications were also reviewed.

\section{MATERIALS AND METHODS}

Between November 2017 and July 2018, patients who were referred for liver biopsy with EUS-FNA were included in the 
study. All patients were 18 years or older and diagnosed with a lesion in the liver by ultrasound, CT, or magnetic resonance imaging. All patients were informed about the procedure and informed consent was obtained. Ethical approval was obtained from the Institutional Review Board (No: 69/2019). Data of the patients were obtained from the hospital registry.

\section{Patient selection}

The selection criteria of the patients for EUS-FNA include the following: (1) patients with lesions suspected as HCC but not showing typical radiological appearances, (2) those with known extrahepatic malignancies (pancreas, colon, etc.) who were referred for confirmation of the metastases, (3) those who had masses in the gallbladder bed, and (4) those who had liver masses. EUS-FNA was planned as the first choice without any concern regarding the difficulty of the lesion access. Antiplatelet medications were discontinued at least 7 days before the procedure. The platelet count had to be above 50,000, and the international normalised ratio value had to be below 1.4 before the procedure. Informed consent was obtained from all patients.

\section{Endoscopic ultrasound and endoscopic ultrasound- guided fine needle aspiration procedure}

EUS-FNA was performed by a single endoscopist with a Fujinon echoendoscope (EG-580 UT; Fujinon, Tokyo, Japan). A 22-G needle was used for FNA (EZ Shot $2^{\mathrm{TM}}$; Olympus Co., Ltd., Tokyo, Japan). Aspirations and biopsies were performed through the duodenum or stomach where the image and access to the lesion were the best. The needle was inserted into the lesion under EUS guidance (Fig. 1, Supplementary Videos 1, 2). After entering the lesion, the stylet was removed. The needle was moved back and forth within the lesion while applying negative pressure with a $10-\mathrm{mL}$ syringe. The needle was moved forward and backward averagely 25-30 times within the lesion. Only a single pass was performed. Aspirated samples were evaluated by a single pathologist. On-site pathologist was not available during the procedures. Prophylactic antibiotherapy was not administered.

\section{Cytological and histological evaluation}

All patients had both air dried and alcohol-fixed preparations. Air dried preparations were stained with May-
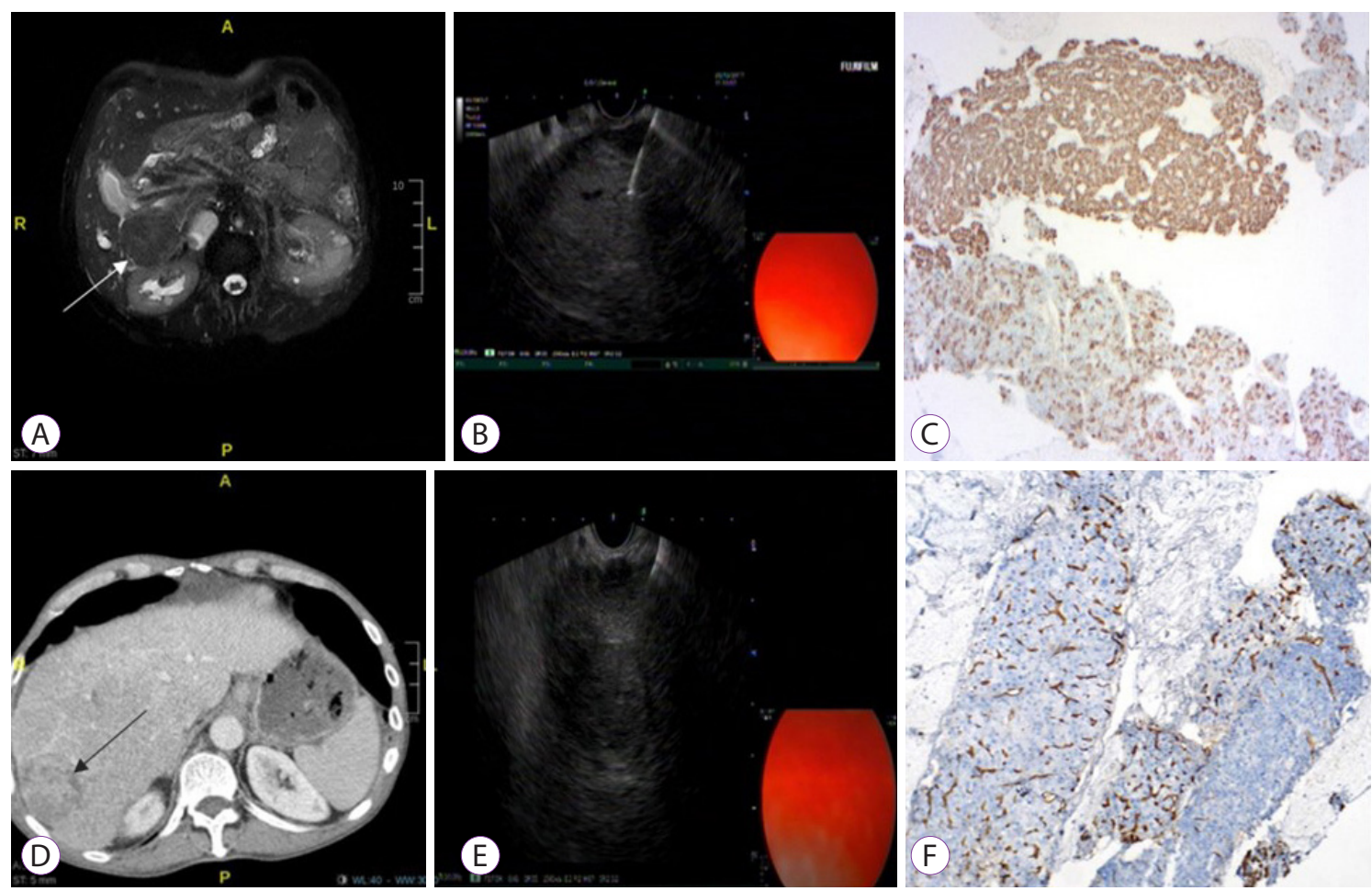

Fig. 1. Performance of endoscopic ultrasound-guided biopsy from lesions in the right liver lobe. (A) A fat-suppressed T2-weighted magnetic resonance image shows an exophytic, isointense liver mass originating from segment 6. (B) An endoscopic ultrasound-guided fine needle aspiration (EUS-FNA) procedure to a $30 \mathrm{~mm}$ hyperechoic mass located in segment 6. (C) Diffusely positive staining is achieved in areas with acinar pattern, and focal staining is noted in thick trabecular areas with HepPar dye $(\times 40)$. (D) Liver lesions in segment 6 , showing intense heterogenous contrast enhancement at the early arterial phase on computed tomography. (E) EUS-FNA procedure to a $25 \mathrm{~mm}$ hyperechoic mass located in segment 6 . (F) The hepatocellular carcinoma tissues located on the left side of the figure show an increase in capillarization unlike the normal parenchyma on the right side $(\mathrm{CD} 34, \times 40)$. 


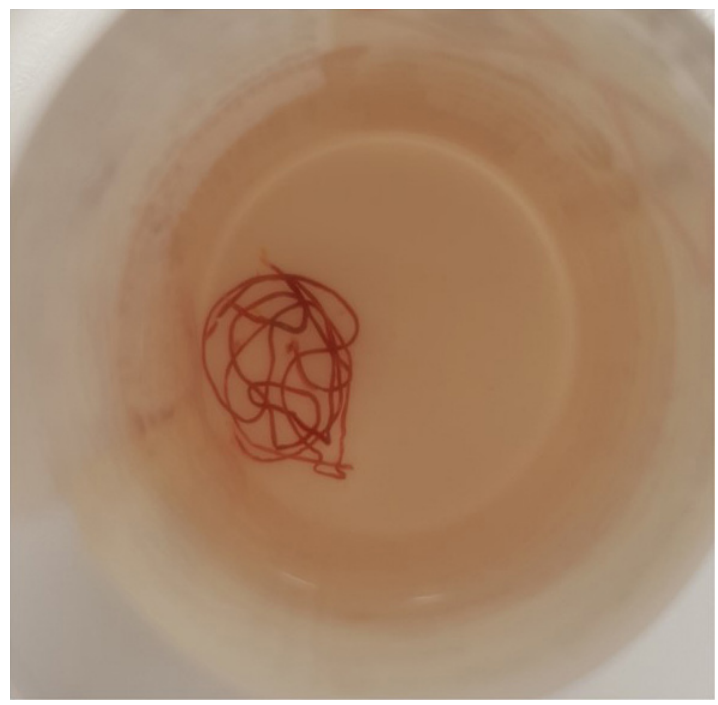

Fig. 2. Macroscopic view of the biopsy material obtained with endoscopic ultrasound guidance.
Grunwald-Giemsa stain and alcohol-fixed preparations were stained by Papanicolaou staining method. The tissue obtained for histological analysis was put in 10\% buffered formalin (Fig. 2). Tissue particles taken together with the biopsy were centrifuged following fixation within the tissue solution. This concentrated and precipitated material was used for liquid-based cytology preparation, and the remaining material was converted into cell blocks.

Formalin-fixed tissues and cell blocks were stored as blocks after routine follow-up in the tissue tracking device. Hematoxylin and eosin (H\&E) staining of sections was performed on the obtained tissues. During the preparation of the sections, for immunohistochemical (IHC)/histochemical tests, a minimum of 5 and a maximum of 10 synchronous sections were prepared on the lysine-coated plates. On average, 8 (range, 1-16) IHC and/or histochemical tests were performed on the obtained tissues.

\section{Histochemical and immunohistochemical evaluation}

Reticulin stain, pCEA, CD34, Glipican 3, and Ki67 IHC markers were utilized for the differential diagnosis of HCC.
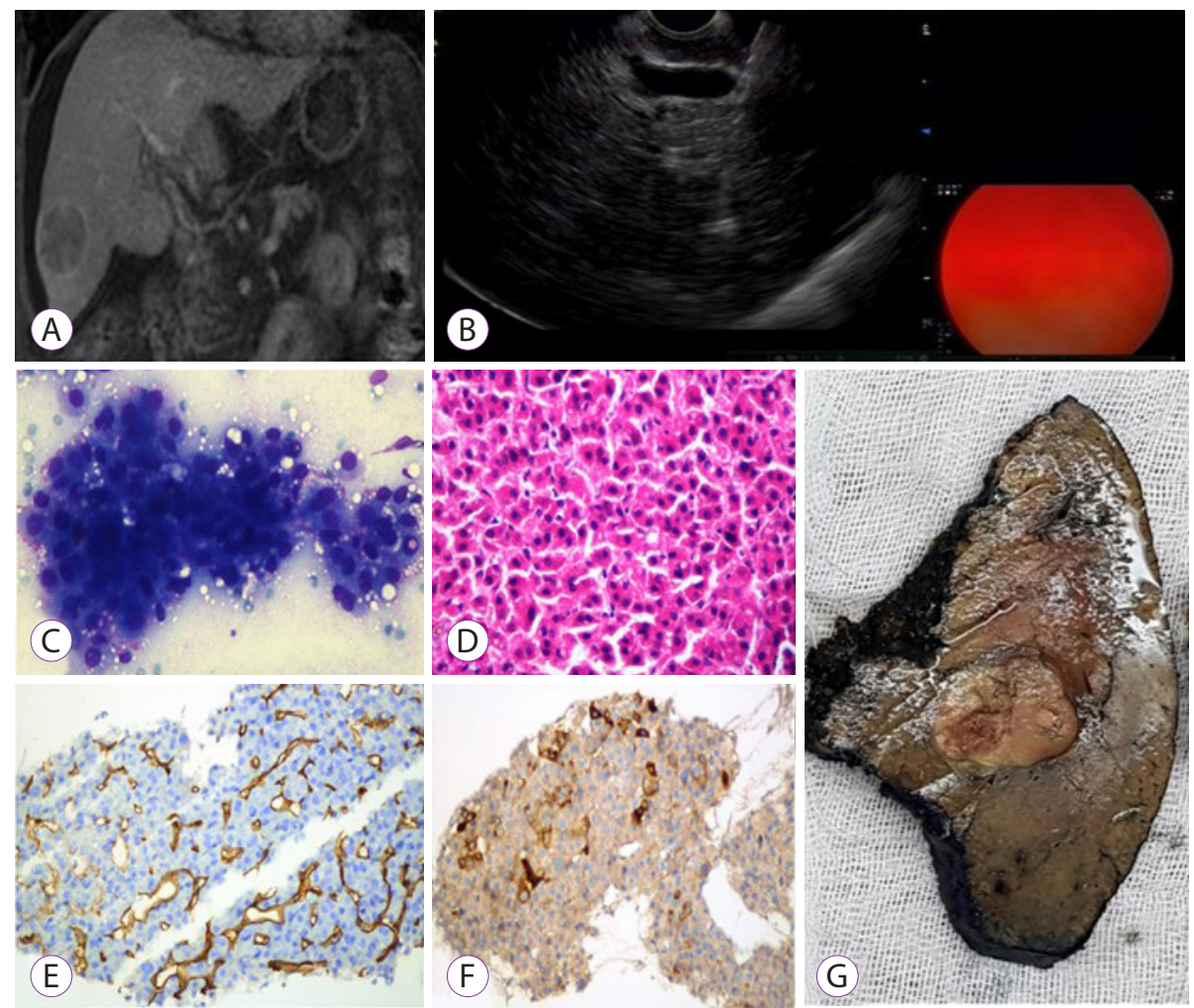

Fig. 3. (A) A well-defined mass with an enhanced capsule in the liver, visualized at the venous phase of a contrast-enhanced, coronal fat-suppressed T1-weighted magnetic resonance image. (B) A $28 \times 34 \mathrm{~mm}$ hypo-/iso-echoic mass in segment 6 during the endoscopic ultrasound-guided fine needle aspiration procedure. (C) Monotonous hepatocellular carcinoma cells stained with May-Grunwald-Giemsa (MGG) in the cytological specimen (MGG, $\times 400)$. (D) Tumor cells stained with hematoxylin and eosin $(\times 200)$. (E) Increased capillarization and thick cellular trabeculae in the biopsy obtained under endoscopic ultrasound (CD34, $\times 200)$. (F) Alfafetoprotein (AFP) positive stained cells in the endoscopic ultrasound biopsy (AFP, $\times 200$ ). (G) Macroscopic appearance of the resected tumor. 

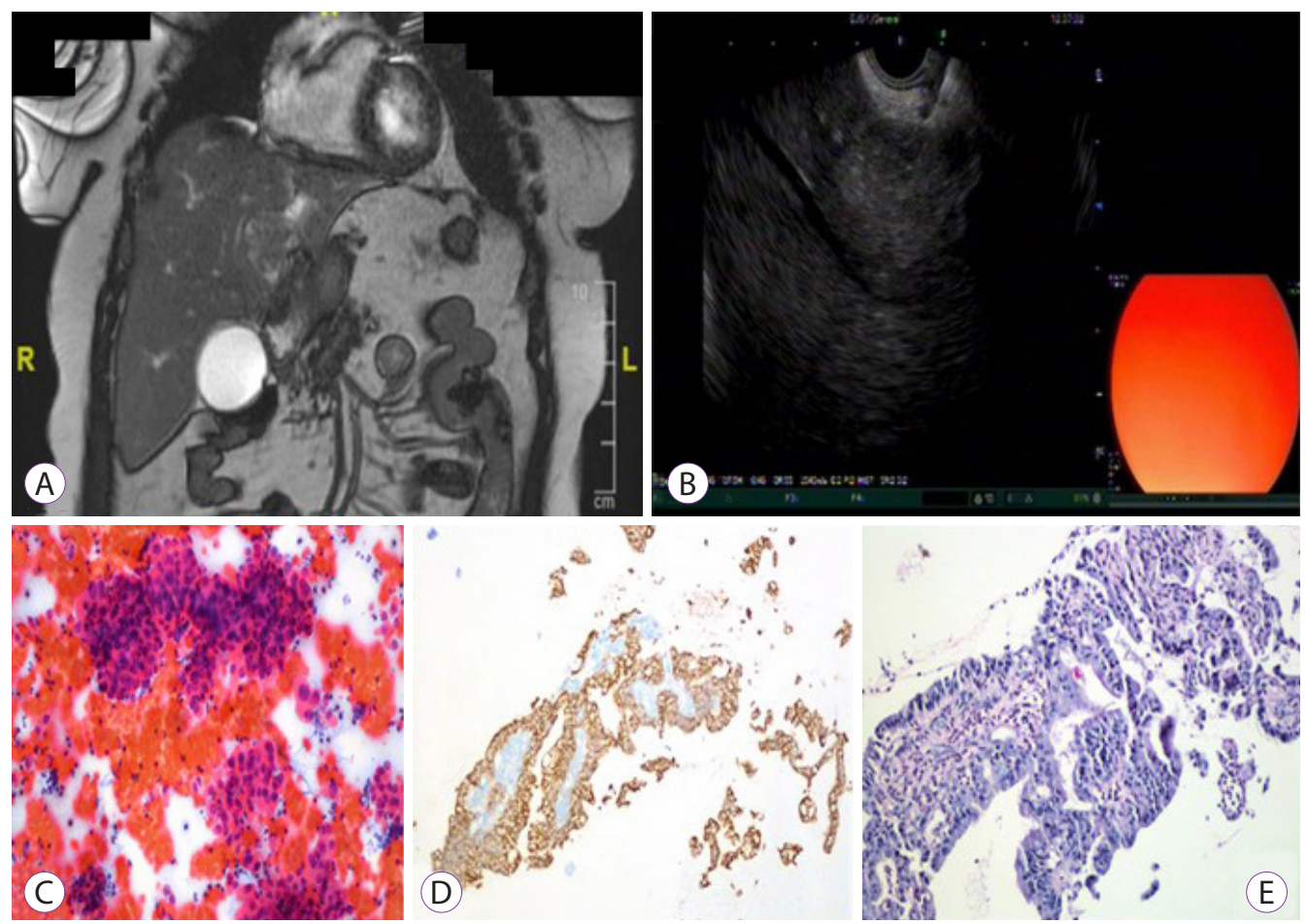

Fig. 4. A cholangiocarcinoma evaluation. (A) An isointense hepatic mass in the left lobe having lobulated contours with infiltrative nature causing segmental intrahepatic bile duct dilatation in coronal T2-weighted 2D fast imaging employing steady-state acquisition (FIESTA) magnetic resonance image. (B) Endoscopic ultrasound-guided fine needle aspiration procedure to a $25 \mathrm{~mm} \times 28 \mathrm{~mm}$ hyperechoic mass located in segment 4. (C) Tumor cells showing 3D groupings with Papanicolaou-stained aspiration ( $\times 200)$. (D) Tumor cells showing adenoid structures with CK7 positive staining in the biopsy material $(\times 100)$. (E) Appearance of the tumor cells with hematoxylin and eosin stain $(\times 200)$.

CK7, CK20, mCEA, HepPar 1, S100, and TTF1 were utilized for the differential diagnosis of adenocarcinoma. Immune markers like Chromogranin A, synaptophysin, and Ki67 were used for the differential diagnosis of neuroendocrine tumors (Figs. 3 and 4).

Following the first phase of staining, an additional one was also performed when required. For the IHC analysis, the XT ultraView DAB procedure was performed for staining in the Ventana BenchMark XT machine. Stained slides were closed with closing solutions after passing through alcohol (increasing grades) and xylene. Preparations were evaluated by a pathologist under a light microscope (Olympus BX53).

\section{Classification of the results}

Cytological and histological diagnoses were classified as follows: nondiagnostic, negative (benign), suspicious for malignancy, and malignancy positive. Cases suspicious for malignancy and malignancy-positive ones were classified as positive. Negative cases were classified as malignancy negative. Either histological or cytological positiveness was enough for consideration as malignancy positive.

In malignancy-positive cases, the definitive diagnoses were made according to surgical pathology results, USG-guided Tru-Cut biopsy results, and other clinicolaboratory findings. In benign lesions, the definitive diagnoses were made according to clinical and radiological findings during the 6th and 12th months of follow-up surveillance. According to these data, the diagnostic accuracy, sensitivity, specificity, positive predictive value (PPV), and negative predictive value (NPV) of EUS-FNA were calculated. As reported previously, the impact of EUS-FNA was calculated considering all of the following: preventing unnecessary surgeries, role in the first diagnosis, upstaging the tumor, and modifying the patient management. ${ }^{1}$

\section{Statistical analysis}

Categorical variables were described as frequency and $n$ (\%). Non-categorical (continuous) variables were described as mean \pm standard deviation. All statistical analyses were calculated using the SPSS version 22.0 (SPSS Inc., Chicago, IL, USA) software. 
Table 1. Characteristics of the Patients with Liver Mass Who Underwent Endoscopic Ultrasound-Guided Fine Needle Aspiration and Biopsy

\begin{tabular}{lc}
\hline Characteristics & $\boldsymbol{n = 2 5}$ \\
\hline Age, yr (mean \pm SD) & $62.73 \pm 15.24$ \\
Sex (Male/Female) & $15 / 10$ \\
Tumor location, $n(\%)$ & \\
$\quad$ Left lobe & \\
$\quad$ S1, S2, S3, S4 & $0 / 3 / 3 / 3$ \\
Right lobe & \\
$\quad$ S5, S6, S7, S8 & $6 / 4 / 2 / 2$ \\
Gallbladder & 2 \\
Platelet count, mean \pm SD & $249 \times 10^{3} \pm 102 \times 10^{3}$ \\
INR & $1.09 \pm 0.11$ \\
\hline
\end{tabular}

INR, international normalised ratio; SD, standard deviation.

\section{RESULTS}

A total of 25 patients who were referred to our center for the evaluation of liver lesions with EUS-FNA were included in the study. Demographics and clinical characteristics of the patients were presented in Table 1. The flowchart for patients included in the study was presented in Fig. 5. Six patients had chronic liver disease due to hepatitis $B$ virus, and one had such disease due to hepatitis $\mathrm{C}$ virus. The median Alfa-fetoprotein level of these patients was $12.46(7.20-42.4) \mathrm{ng} / \mathrm{mL}$ (IQR, 25-75). Three patients had ascites due to cirrhosis.

Biopsies were performed technically successfully in 22 (88\%) patients. Of these, 11 (50\%) were performed through the stomach, and the remaining $11(50 \%)$ were performed through the duodenum. The lesion could not be localized

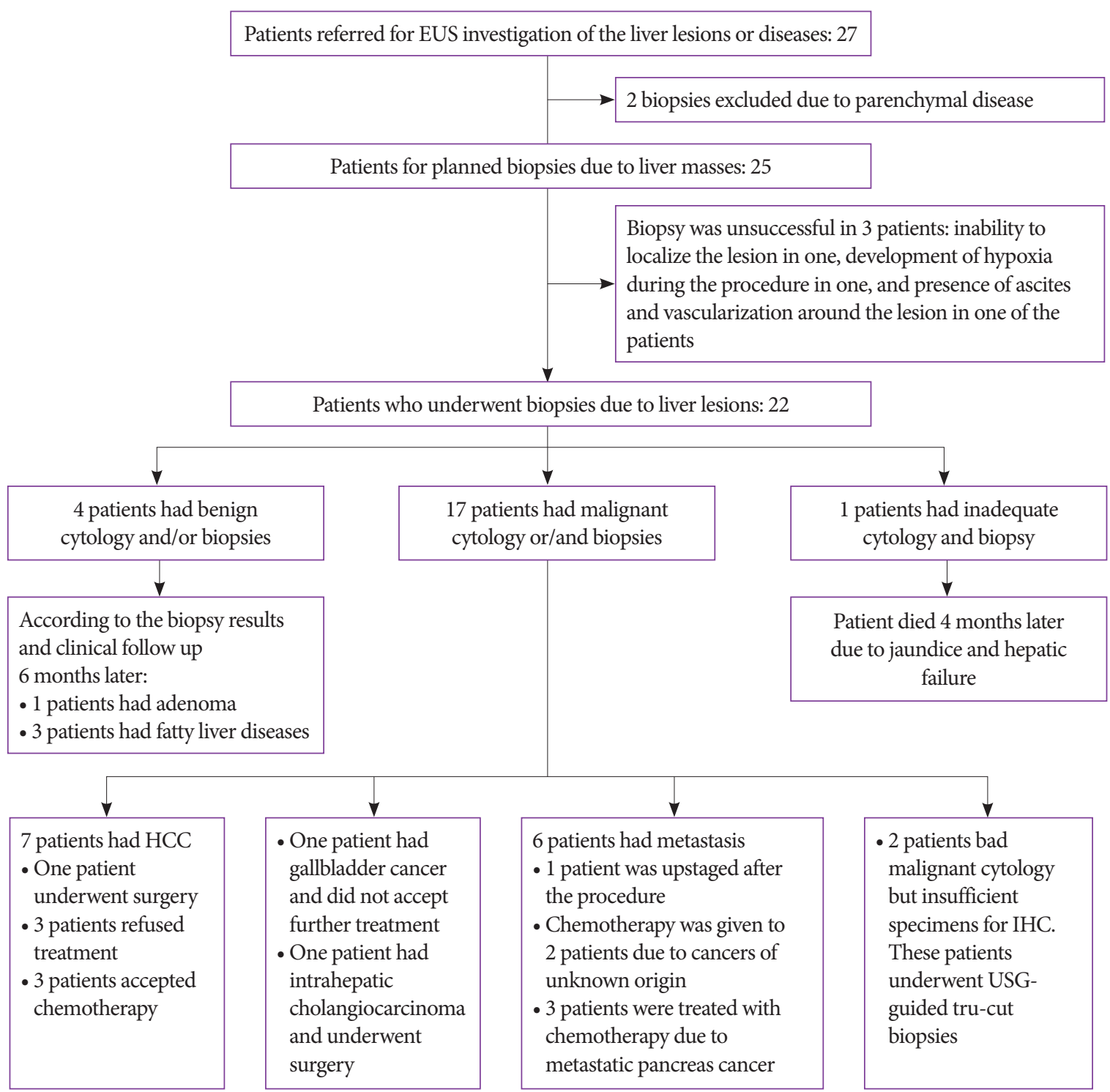

Fig. 5. Flowchart of the patients with hepatic lesions. EUS, endoscopic ultrasound; HCC, hepatocellular carcinoma; IHC, immunohistochemical; USG, ultrasonography 
Table 2. Endoscopic Ultrasound Features and Fine Needle Aspiration Biopsy Results according to the Final Diagnoses in 22 Patients Who Underwent Endoscopic Ultrasound-Guided Fine Needle Aspiration

\begin{tabular}{|c|c|c|c|c|}
\hline \multirow{3}{*}{ Characteristics } & \multicolumn{4}{|c|}{ Final diagnosis $(n)$} \\
\hline & HCC & Extrahepatic origin of the mass & Cholangiocarcinoma & Benign \\
\hline & $n=7$ & $n=10$ & $n=1$ & $n=4$ \\
\hline \multicolumn{5}{|l|}{ Tumor characteristics } \\
\hline Size, $\mathrm{mm}$ & $44.29 \pm 6.31$ & $34.20 \pm 18.55$ & $25 \times 28$ & $24.25 \pm 11.14$ \\
\hline Location, $\mathrm{Sn}(n)$ & $\begin{array}{l}\text { S3 (1), S5 (2), } \\
\text { S6 (3), S7 (1) }\end{array}$ & $\begin{array}{l}\text { S2 (2), S3 (1), S5 (4), } \\
\text { S6 (1), gallbladder (2) }\end{array}$ & S4 (1) & $\begin{array}{l}\mathrm{S} 2(1), \mathrm{S} 3(1) \\
\quad \mathrm{S} 4(2)\end{array}$ \\
\hline \multicolumn{5}{|l|}{ Puncture route } \\
\hline Transgastric & 1 & 5 & 1 & 4 \\
\hline Transduodenal & 6 & 5 & 0 & 0 \\
\hline \multicolumn{5}{|l|}{ Echogenicity } \\
\hline Hypoechoic & 0 & 6 & 0 & 0 \\
\hline Hyperechoic & 0 & 2 & 1 & 4 \\
\hline Mixed echoic & 7 & 2 & 0 & 0 \\
\hline \multicolumn{5}{|l|}{ Border } \\
\hline Regular & 0 & 6 & 0 & 2 \\
\hline Irregular & 7 & 4 & 1 & 2 \\
\hline \multicolumn{5}{|l|}{ Lymphadenopathy } \\
\hline Yes/No & $0 / 7$ & $5 / 5$ & 0 & 0 \\
\hline $\begin{array}{l}\text { Adjacent to vascular/ } \\
\text { or compressing biliary channels }\end{array}$ & 5 & 2 & 1 & 0 \\
\hline Postacoustic shadowing & 5 & 2 & 0 & 0 \\
\hline Ascites & 3 & 0 & 0 & 0 \\
\hline FNAB results $(n)$ & $\begin{array}{l}\text { Hepatocellular } \\
\text { cancer }(7)\end{array}$ & $\begin{array}{l}\text { Pancreaticobiliary cancer with liver } \\
\text { metastasis (4) } \\
\text { Colon cancer with liver metastasis (1) } \\
\text { Lung cancer with liver metastasis (1) } \\
\text { Gallbladder cancer with liver infiltration } \\
\text { (1) } \\
\text { Inadequate material (3) }\end{array}$ & Cholangiocarcinoma (1) & $\begin{array}{l}\text { Steatosis (3) } \\
\text { Hepatic adenoma (1) }\end{array}$ \\
\hline
\end{tabular}

The diagnosis was made according to the surgical pathology results in 2 patients, according to ultrasonography-guided tru-cut biopsy results in 2 patients, and according to other clinical and laboratory data in 13 patients. Remaining 5 patients were diagnosed according to imaging studies and clinical follow-up.

FNAB, fine needle aspiration biopsy; HCC, hepatocellular carcinoma, Sn, liver segment number.

in the right posterior lobe in one patient, the EUS procedure was stopped due to hypoxia in one patient, and biopsy was unsuccessful due to the vascularization around the lesion and presence of ascites in one patient. The features of the EUS evaluations are shown in Table 2.

The mean tumor size was calculated as $34.50 \pm 16.04 \mathrm{~mm}$. Seven patients had more than one lesion, and 15 had single lesions. Of the 22 patients, 21 (95.45\%) had sufficient aspirations, and 19 (86.3\%) had sufficient biopsies for the H\&E and IHC tests. Two (9\%) patients had aspiration results consistent with malignancy; however, biopsies were insufficient for further analysis. These patients had the final diagnoses of linitis plastica and neuroendocrine tumor metastasis after USG-guided Tru-Cut biopsies. One (4.5\%) patient had insufficient aspiration and biopsy. During the follow-up of this patient, the lesion in the gallbladder bed showed fast radiological progression leading to mechanical icterus and thus was diagnosed as gallbladder cancer. The patient was lost to fol- 
Table 3. Overall Technical Outcomes of Fine Needle Aspirations and Biopsies

\begin{tabular}{lc}
\hline Characteristics & $\boldsymbol{n = 2 2 ( \% )}$ \\
\hline Adequate specimen & \\
Cytology with PAP/ MGG & $21(95.4)$ \\
Histology with H\&E & $19(86.3)$ \\
Histology with IHC & $19(86.3)$ \\
Diagnostic categories & \\
Non-diagnostic & $1(4.5)$ \\
Benign & $4(18.2)$ \\
Suspicious for malignancy & $1(5.5)$ \\
Positive for malignancy & $16(72.8)$ \\
Diagnostic accuracy for malignancy & $17 / 18(94.4)^{\mathrm{a})}$ \\
Diagnostic accuracy for specific tumor & $16 / 19(84.2)^{\mathrm{b})}$ \\
Complications, $n$ & $0(0)$ \\
\hline
\end{tabular}

H\&E, hematoxylin and eosin; IHC, immunohistochemical; MGG, May-Grunwald-Giemsa; PAP, Papanicolaou.

a) This value was calculated according to malignant tumors. ${ }^{\text {b) For }}$ specific tumors, the values were calculated according to the malignant and benign tumors.

low-up at 4 months following the biopsy. The overall success rates of EUS-FNA and biopsies are listed in Table 3. The final diagnoses of hepatic lesions and methods of the diagnoses are summarized in Table 2.

Among patients with malignant cytology, EUS-FNA aspiration had clinical impact (according to definition) in $17(100 \%)$ patients. EUS-FNA helped in the final diagnoses of $15(83.3 \%)$ patients with malignant cytology. Out of 15 patients, 7 (46.7\%) were diagnosed with HCC, one (6.7\%) with gallbladder cancer, one $(6.7 \%)$ with cholangiocarcinoma, and six (40\%) with metastases. EUS-FNA helped in the diagnosis of liver metastasis in three (17.6\%) patients, and these patients were referred for chemotherapy, thus avoiding surgery. Two (11.7\%) patients were referred for surgery. Five (29.4\%) patients (three HCC with ascites and two adenocarcinomas with unknown origin) were referred for chemotherapy due to inoperability.

Of the remaining five patients, four (three HCC and one gallbladder cancer) rejected the treatment, and one (lung adenocarcinoma) had upstaging. Overall, management was modified in 10 (59\%) patients. Tru-Cut biopsy was needed in two patients (metastases) for the final diagnoses. EUS-FNA had sensitivity, specificity, NPV, and PPV rates of $94.4 \%, 100 \%$, $80 \%$, and $100 \%$, respectively. The diagnostic accuracy for all lesions was calculated as $86.3 \%(19 / 22)$. There were no complications after the procedure such as bleeding or infection.

\section{DISCUSSION}

EUS-FNA has appeared as a key tool for the diagnostic evaluation, staging, and treatment of gastrointestinal tract lesions. In this study, we used EUS-FNA in liver lesions that were difficult to reach with conventional techniques and those with a high risk of complications. Through single pass, aspiration and biopsy sufficiencies were $95.4 \%$ and $86.3 \%$, respectively. We did not encounter any complications. Overall, EUS-FNA modified the management of 10 (59\%) patients.

Both primary liver lesions and liver metastases may have important impacts on the management and prognosis of the patients. CT is a standard imaging modality in the detection of liver masses. EUS is a well-described test for the diagnosis and staging of gastrointestinal and lung tumors and has become an alternative tool for liver imaging. ${ }^{8}$ Nguyen et al. were the first to report that CT was able to demonstrate only $21 \%$ of the liver lesions detected by EUS. ${ }^{9}$ They showed that EUS could detect liver metastases that could not be detected by CT and this could change the $\mathrm{M}$ stage of a disease and its management. ${ }^{9}$ Awad et al. reported that EUS investigation and FNA from the liver lesions changed the management in $67 \%$ of the patients. ${ }^{4}$ Dewitt et al. reported $86 \%$ change after EUS-FNA in the management of solid liver lesions in 77 patients. ${ }^{1}$ In our series, this ratio was $59 \%$. Rejection of the treatment by four of our patients may have caused the decreased rate. When four patients who rejected the treatment were added to the calculation, the impact on the patient management rate increased to $82 \%$.

Whether ultrasonographic features of the lesions that are evaluated by EUS could predict if they are benign or malignant is of utmost importance. tenBerge et al. reported that the shape, size, echogenicity, and features of the borders of the lesions were not predictable in the differentiation between benign and malignant lesions. ${ }^{10}$ Fujii-Lau et al. developed a scoring system according to the endosonographic features with $88 \%$ PPV rate and reported that this could help in deciding on which lesions should be biopsied or not. ${ }^{11}$ In this series, we saw that geographically shaped lesions were benign and malignant lesions had more surrounding vascular and biliary channel distortions and greater post-acoustic shadowing. Moreover, the echogenicity and border features of the lesions were not helpful in the differentiation between benign and malignant lesions (Table 2). Therefore, we suggest that once a lesion is demonstrated, FNA should be performed regardless of its echogenicity.

The diagnostic accuracy of EUS-FNA in hepatic masses was reported between $82 \%$ and $100 \% .{ }^{12}$ Lee and colleagues reported $90.5 \%$ and $86.3 \%$ diagnostic yields after core needle biopsies for liver lesions, in malignant and specific tumors, re- 
spectively. ${ }^{13}$ In our study, the diagnostic yield rate for all lesions was calculated as $86.3 \%$. In a prospective study carried out by Hollerbach et al., the sensitivity, specificity, NPV, and PPV rates were $94 \%, 100 \%, 78 \%$, and $100 \%$, respectively. ${ }^{14}$ In this study, these rates were $94.4 \%, 100 \%, 80 \%$, and $100 \%$, respectively.

In our study, with single pass, we obtained sufficient aspirates in $95.4 \%$ and sufficient biopsies for necessary stainings in $86.3 \%$ of the patients. Nguyen et al. reported a mean of two passes and diagnostic accuracy of $100 \%{ }^{9}$ Moreover, Oh et al. reported a mean pass number of 3 , technical success rate of $97.9 \%$, and sufficient aspirate rate of $91.3 \% .^{15}$ To achieve an acceptable success rate with one pass, we performed 25-30 times to-and-fro movements within the lesion. During the procedure, we did not have an on-site cytopathologist. It could be proposed that reduced pass numbers may shorten the procedure duration, decrease complications like bleeding, and reduce seeding risk.

The most challenging parts of the liver to visualize during EUS are the right posterior and anterosuperior segments. ${ }^{10}$ The gallbladder and portal vascular structures interfere with the vision during the procedure in these regions. Oh et al. compared the right and left lobe EUS-FNA procedures and reported diagnostic yield rates of $89.3 \%$ and $92.9 \%$, respectively. ${ }^{15}$ In one patient, the lesion was not able to localize in the right posterior lobe. In our series, the lesion was not visualized in one patient due to its localization in the posterior part of the right lobe. By increasing the tissue depth with low frequencies (5 Mhz), the problems with transbulbar and transduodenal approach could be overcome. Despite this limitation, EUSFNA has some advantages over the percutaneous USG-guided needle biopsy technique, including less influence from the respiration, performance in less cooperative patients, performance in obese patients and those with massive ascites, and ease in approaching the caudate lobe. In addition, compared with USG-guided percutaneous biopsies, less post-procedural pain was reported in EUS-guided liver biopsies. ${ }^{16}$

As the experience with EUS-guided liver biopsy increases, newly designed needles for this procedure emerge. Eskandari et al. compared needles with different geometric tips and sizes of 19-, 20-, 22-G in the fine needle aspiration biopsy (FNAB) procedure in a freshly harvested bovine liver and showed that 19-G needles had higher diagnostic performance compared with $20-\mathrm{G}$ needles. ${ }^{17}$ In a randomized study with cadaveric livers, Schulman et al. reported that biopsies with $22-\mathrm{G}$ needles had higher numbers of portal tracts compared with those with $19-\mathrm{G}$ needles. ${ }^{18}$ Because either of the studies was not performed in real patients, it is not possible to obtain any information about the adverse events. In this study, with the help of EZ Shot 2 22-G needle, we succeeded to achieve a higher amount of FNAB material. The presence of side hole in its specifically designed tip may have helped in our high yield.

The major complication rates with 22- or $24-\mathrm{G}$ needles were reported to be between $0 \%$ and $4 \% .{ }^{12}$ Major complications include infection, hemorrhage, biliary peritonitis, and rarely malignant cell seeding. ${ }^{19}$ In a retrospective series with $167 \mathrm{pa}$ tients, the major complication rate of EUS-FNA in hepatic lesions was reported to be approximately $1 \%$. In this series, there were two patients with abdominal pain and two with fever, and one patient died. ${ }^{10}$ In a meta-analysis, it was reported that the EUS-FNA procedure had a greater risk in the liver than in other localizations, and this risk increased with ascites. ${ }^{20}$ In this series, we did not encounter any complications. We had three cirrhotic patients with ascites, and their platelet count was between 50,000 and 70,000. These results were in favor of the safety of the EUS-FNA procedure for liver biopsy.

The main limitations of this study were its retrospective design and limited sample size. However, the presence of the same specialists during both EUS procedure and pathological investigations could be its strengths. Increased successful biopsy rates accompanying the increased successful cytology rates further enriched our results. Several types of liver lesions, both benign and malignant, were included in this study.

In summary, EUS-FNA for liver lesions have high success rates in tissue sample retrieval and lower complication rates even in patients with ascites. This procedure should be considered for liver lesions that are hard to reach with the conventional methods or bearing high complication risks.

\section{Conflicts of Interest}

The authors have no potential conflicts of interest.

Funding

None.

ORCID
Ebru Akay:

Deniz Atasoy:

Engin Altınkaya:

Ali Koç:

Tamer Ertan:

Hatice Karaman:

Erkan Caglar: https://orcid.org/0000-0003-1190-1800 https://orcid.org/0000-0002-5703-4549 https://orcid.org/0000-0001-6636-7073 https://orcid.org/0000-0003-0296-4914 https://orcid.org/0000-0003-3721-2253 https://orcid.org/0000-0002-5250-5663 https://orcid.org/0000-0001-7246-0952

\section{Supplementary Materials}

Video 1. Endoscopic ultrasound-guided fine needle aspiration procedure from the lesion in the right lobe (https://doi.org/10.5946/ce.2020.065. v001).

Video 2. Endoscopic ultrasound-guided fine needle aspiration procedure from the lesion in the left lobe (https://doi.org/10.5946/ce.2020.065. v002). 


\section{REFERENCES}

1. Dewitt J, McGreevy K, Cummings $\mathrm{O}$, et al. Initial experience with EUS-guided Tru-cut biopsy of benign liver disease. Gastrointest Endosc 2009;69:535-542.

2. Hammoud GM, Almashhrawi A, Ibdah JA. Usefulness of endoscopic ultrasound-guided fine needle aspiration in the diagnosis of hepatic, gallbladder and biliary tract lesions. World J Gastrointest Oncol 2014;6:420429

3. Parekh PJ, Majithia R, Diehl DL, Baron TH. Endoscopic ultrasound-guided liver biopsy. Endosc Ultrasound 2015;4:85-91.

4. Awad SS, Fagan S, Abudayyeh S, Karim N, Berger DH, Ayub K. Preoperative evaluation of hepatic lesions for the staging of hepatocellular and metastatic liver carcinoma using endoscopic ultrasonography. Am J Surg 2002;184:601-604; discussion 604-605.

5. Mathew A. EUS-guided routine liver biopsy in selected patients. Am J Gastroenterol 2007;102:2354-2355.

6. Gleeson FC, Clayton AC, Zhang L, et al. Adequacy of endoscopic ultrasound core needle biopsy specimen of nonmalignant hepatic parenchymal disease. Clin Gastroenterol Hepatol 2008;6:1437-1440.

7. Stavropoulos SN, Im GY, Jlayer Z, et al. High yield of same-session EUS-guided liver biopsy by 19-gauge FNA needle in patients undergoing EUS to exclude biliary obstruction. Gastrointest Endosc 2012;75:310-318.

8. Tharian B, Tsiopoulos F, George N, Pietro SD, Attili F, Larghi A. Endoscopic ultrasound fine needle aspiration: technique and applications in clinical practice. World J Gastrointest Endosc 2012;4:532-544.

9. Nguyen P, Feng JC, Chang KJ. Endoscopic ultrasound (EUS) and EUS-guided fine-needle aspiration (FNA) of liver lesions. Gastrointest Endosc 1999;50:357-361.

10. tenBerge J, Hoffman BJ, Hawes RH, et al. EUS-guided fine needle aspiration of the liver: indications, yield, and safety based on an international survey of 167 cases. Gastrointest Endosc 2002;55:859-862.
11. Fujii-Lau LL, Abu Dayyeh BK, Bruno MJ, et al. EUS-derived criteria for distinguishing benign from malignant metastatic solid hepatic masses. Gastrointest Endosc 2015;81:1188-1196.e1-e7.

12. Ichim VA, Chira RI, Mircea PA. Diagnostic yield of endoscopic ultrasound-guided biopsy of focal liver lesions. Med Pharm Rep 2019;92:1520

13. Lee YN, Moon JH, Kim HK, et al. Usefulness of endoscopic ultrasound-guided sampling using core biopsy needle as a percutaneous biopsy rescue for diagnosis of solid liver mass: combined histological-cytological analysis. J Gastroenterol Hepatol 2015;30:1161-1166.

14. Hollerbach S, Reiser M, Topalidis T, König M, Schmiegel W. Diagnosis of hepatocellular carcinoma (HCC) in a high-risk patient by using transgastric EUS-guided fine-needle biopsy (EUS-FNA). Z Gastroenterol 2003;41:995-998.

15. Oh D, Seo DW, Hong SM, et al. Endoscopic ultrasound-guided fine-needle aspiration can target right liver mass. Endosc Ultrasound 2017;6:109-115.

16. Chon HK, Yang HC, Choi KH, Kim TH. Endoscopic ultrasound-guided liver biopsy using a core needle for hepatic solid mass. Clin Endosc 2019;52:340-346.

17. Eskandari A, Koo P, Bang H, Gui D, Urayama S. Comparison of endoscopic ultrasound biopsy needles for endoscopic ultrasound-guided liver biopsy. Clin Endosc 2019;52:347-352.

18. Schulman AR, Thompson CC, Odze R, Chan WW, Ryou M. Optimizing EUS-guided liver biopsy sampling: comprehensive assessment of needle types and tissue acquisition techniques. Gastrointest Endosc 2017;85:419-426.

19. Jovani M, Abidi WM, Lee LS. Novel fork-tip needles versus standard needles for EUS-guided tissue acquisition from solid masses of the upper GI tract: a matched cohort study. Scand J Gastroenterol 2017;52:784787.

20. Wang KX, Ben QW, Jin ZD, et al. Assessment of morbidity and mortality associated with EUS-guided FNA: a systematic review. Gastrointest Endosc 2011;73:283-290 